\title{
Analytic Moduli Spaces as Orbit Spaces
}

\author{
By \\ Jürgen BINGENER*
}

\section{Introduction}

In this paper we formulate an abstract quotient theorem for convergent maps of PO-spaces (4.3), being inspired by the work [6] of I.F. Donin. This theorem, which is proved in detail in [5], gives a criterion for certain quotient groupoids over the category of germs of analytic spaces to have a semiuniversal deformation. With this result in hands, one can solve many local moduli problems in analytic geometry in a unified manner, see $[3,4]$ and [5]. Let us finally remark that we use the notions and notations introduced in [2] without any further comment.

\section{$\S 1$. Formal Maps}

1.1. In the following $K$ denotes a fixed commutative Q-algebra. For a $K$-module $F$ and finite family $E_{i}, i \in I$, of $K$-modules the $K$-multilinear maps $\varphi: \Pi_{i \in I} E_{i} \rightarrow F$ form a $K$-module $\operatorname{Mult}_{K}\left(E_{i}, i \in I ; F\right)$. In case $I=\{1, \cdots, p\}$ we write $\operatorname{Mult}_{K}\left(E_{1}, \cdots, E_{p} ; F\right)$ instead of $\operatorname{Mult}_{K}\left(E_{i}, i \in I ; F\right)$.

For two $K$-modules $E$ and $F$ and $p \in \mathbb{N}$ let $\operatorname{Mult}_{p}(E, F)=\operatorname{Mult}_{K, p}(E, F)$ denote the $K$-module $\operatorname{Mult}_{K}\left(E_{1}, \cdots, E_{p} ; F\right)$ with $E_{\imath}:=E$ for $1 \leqq i \leqq p$, and let $\operatorname{Hom}_{p}(E, F)=\operatorname{Hom}_{K, p}(E, F)$ be the image of the $K$-linear map $\operatorname{Mult}_{p}(E, F) \rightarrow F^{E}$ sending $\varphi$ to $\varphi \Delta^{(p)}$; here $\Delta^{(p)}: E \rightarrow E^{p}$ is the diagonal map. The elements of the $K$-module $F \llbracket E \rrbracket:=F_{K} \llbracket E \rrbracket:=\prod_{p \in N} \operatorname{Hom}_{p}(E, F)$ are called formal power series on $E$ with values in $F$. For a power series $u=\sum_{p \in N} u_{p}$ from $F \llbracket E \rrbracket$ we denote by $\mathrm{T}(u):=u_{1}$ the tangent map of $u$. Let $F \llbracket E \rrbracket_{+}$be the submodule of $F \llbracket E \rrbracket$ consisting of all power series without constant term.

Suppose now that $E$ is of the form $E=E_{1} \times \cdots \times E_{k}$ with $K$-modules $E_{1}, \cdots, E_{k}$. For an element $\nu=\left(\nu_{1}, \cdots, \nu_{k}\right)$ of $N^{k}$ we put $E_{\nu}:=E_{1}^{\nu_{1}} \times \cdots \times E_{k}^{\nu^{k}}$ for abbreviation, and denote by $\operatorname{Mult}_{\nu}(E, F)=\operatorname{Mult}_{K, \nu}(E, F)$ the $K$-module consisting

Communicated by M. Kashiwara, November 26, 1986.

* Fakultät für Mathematik der Universität, D-8400 Regensburg, Federal Republic of Germany. 
of all $|\nu|$-linear maps $\varphi: E_{\nu} \rightarrow F$. Further let $\operatorname{Hom}_{\nu}(E, F)=\operatorname{Hom}_{K, \nu}(E, F)$ be the image of the $K$-linear map $\operatorname{Mult}_{\nu}(E, F) \rightarrow F^{E}$ sending $\varphi$ to $\varphi \Delta^{(\nu)}$; here $\Delta^{(\nu)}: E \rightarrow E_{2}$ is the "multidiagonal" map. Then every formal power series $u$ from $F \llbracket E \rrbracket$ has a unique representation $u=\sum_{\nu \in N^{k}} u_{\nu}$ with elements $u_{\nu}$ of $\operatorname{Hom}_{\nu}(E, F)$.

1.2. By a punctured $K$-module we understand a pair $(E, 0)$ consisting of a $K$-module $E$ and its origin. Let $(E, 0)$ and $(F, 0)$ be two punctured $K$-modules. A formal map $u:(E, 0) \rightarrow(F, 0)$ is a formal power series from $F \llbracket E \rrbracket+$.

Endowed with the formal maps as morphisms (and the composition of formal power series as composition of morphisms), the punctured $K$-modules form a category $\left(\right.$ For $\left._{K}\right)$ with products. For a punctured $K$-module $(E, 0)$ we denote by $(E, 0)^{\circ}$ the corresponding contravariant set valued functor on $\left(\right.$ For $\left._{K}\right)$. Then a formal map $u$ from $(E, 0)$ to $(F, 0)$ induces a morphism of functors from $(E, 0)^{\circ}$ to $(F, 0)^{\circ}$ being denoted by $i$.

1.3. A formal group over $K$ is a punctured $K$-module $G=(G, 0)$ endowed with formal maps $m_{G}: G \times G \rightarrow G$ and $j_{G}: G \rightarrow G$ such that $\dot{G}$ is a group valued functor with respect to $\dot{m}_{G}: \dot{G} \times \dot{G} \rightarrow \dot{G}$, the inversion mapping being $\dot{j}_{G}$.

Let $G$ be a formal group over $K$ and let $(E, 0)$ be a punctured $K$-module. An operation of $G$ on $(E, 0)$ is a formal map $\omega: G \times(E, 0) \rightarrow(E, 0)$ such that $\dot{\omega}$ is an operation of the group valued functor $\dot{G}$ on the set valued functor $(E, 0)$. Then the composition of $\omega$ and $\left(\operatorname{id}_{G}, 0\right): G \rightarrow G \times(E, 0)$ is called the orbital map of $\omega$.

Let $(E, 0)$ and $(F, 0)$ be two punctured $K$-modules, on which $G$ operates. A formal map $u:(E, 0) \rightarrow(F, 0)$ is said to be G-equivariant, if $i$ is equivariant with respect to the action of $\dot{G}$. We say that $G$ operates with fixed point on $(F, 0)$, if the canonical map $0 \rightarrow(F, 0)$ is $G$-equivariant.

\section{$\S 2$. Convergent Maps of PO-Spaces}

2.1. Let $E$ be a $C$-vector space. A pseudo-norm on $E$ is a mapping $\|\cdot\|: E \rightarrow \boldsymbol{R}_{+} \cup\{+\infty\}$ with the following properties: (1) For any $\alpha$ from $C$ and any $x$ from $E$ such that $\|x\|<\infty$ we have $\|\alpha x\|=|\alpha|\|x\|$. (2) $\|x+y\| \leqq\|x\|+\|y\|$ for any pair $x, y$ of elements of $E$.

2.2. Let now $K$ be a commutative $C$-algebra and let $E, F$ be two $K$-modules. We suppose that for every integer $p \in N$ we are given a pseudo-norm $|\cdot|=|\cdot|_{p}$ resp. $\|\cdot\|=\|\cdot\|_{p}$ on $\operatorname{Mult}_{p}(E, F)=\operatorname{Mult}_{K, p}(E, F) \operatorname{resp.} \operatorname{Hom}_{p}(E, F)=\operatorname{Hom}_{K, p}(E, F)$ such that $|\cdot|_{p}=\|\cdot\|_{p}$ for $p=0,1$. If $t>0$ is a real number and $u=\Sigma_{p} u_{p}$ a formal power series from $F \llbracket E \rrbracket$, we put 


$$
|u|_{t}:=\sum_{p \in N}\left|\tilde{u}_{p}\right| t^{p}
$$

and

$$
\|u\|_{t}:=\sum_{p \in N}\left\|u_{p}\right\| t^{p}
$$

here $\tilde{u}_{p}$ denotes the symmetric $p$-lirear map corresponding to $u_{p}$. We say that $u$ is convergent with respect to $\|\cdot\|$ resp. strongly convergent with respect to $|\cdot|$, if there exists a $t>0$ such that $\|u\|_{t}<\infty$ resp. $|u|_{t}<\infty$. Clearly $|\cdot|_{t}$ and $\|\cdot\|_{t}$ are pseudo-norms on $F \llbracket E \rrbracket$ for every $t>0$.

2.3. Let $G$ be another $K$-module and suppose moreover that for every integer $p \in N$ we are given a pseudo-norm $|\cdot|=|\cdot|_{p}$ on $\operatorname{Mult}_{p}(F . G)$ and $\operatorname{Mult}_{p}(E, G)$ and a pseudo-norm $\|\cdot\|=\|\cdot\|_{p}$ on $\operatorname{Hom}_{p}(F, G)$ and $\operatorname{Hom}_{p}(E, G)$ such that $|\cdot|_{p}=$ $\|\cdot\|_{p}$ for $p=0,1$. Furthermore we assume that there exists a real number $\alpha \geqq 1$ with the following property: If $\phi$ is an element of $\operatorname{Mult}_{p}(F, G)$ such that $|\psi|$ $<\infty$ and if $u_{\imath} \in \operatorname{Hom}_{q_{\imath}}(E, F)$ and $\varphi_{\imath} \in \operatorname{Mult}_{q_{\imath}}(E, F)$ are elements such that $\left\|u_{\imath}\right\|$ $<\infty,\left|\varphi_{\imath}\right|<\infty, 1 \leqq i \leqq p$, then in case $p \geqq 1$ and $q_{\imath} \geqq 1$ the estimates

$$
\begin{aligned}
& \left\|\psi \circ\left(u_{1}, \cdots, u_{p}\right)\right\| \leqq \alpha^{q_{1}+\cdots+q_{p}-1}|\psi|\left\|u_{1}\right\| \cdots\left\|u_{p}\right\|, \\
& \left|\psi \circ\left(\varphi_{1} \times \cdots \times \varphi_{p}\right)\right| \leqq \alpha^{q_{1}+\cdots+q_{p}-1}|\psi|\left|\varphi_{1}\right| \cdots\left|\varphi_{p}\right|
\end{aligned}
$$

hold. The following (easy) proposition shows in particular, that in these circumstances the convergence notion introduced in 2.2 is stable under composition.

Lemma 2.4. Let the assumptions and notations be as in 2.3. Further let $u$ resp. $v$ be a formal power series from $F \llbracket E \rrbracket_{+}$resp. $G \llbracket F \rrbracket$ and let $t, s>0$ be real numbers. Then:

(1) In case $\|u\|_{a t} \leqq s$ we have $\|v u\|_{t} \leqq|v|_{s}$.

(2) In case $|u|_{a t} \leqq s$ we have $|v u|_{t} \leqq|v|_{s}$.

Example 2.5. Let $E=(E,\|\cdot\|)$ and $F=(F,\|\cdot\|)$ be two pseudo-normed $K$ modules. For an element $\varphi$ resp. $u$ of $\operatorname{Mult}_{p}(E, F) \operatorname{resp.} \operatorname{Hom}_{p}(E, F)$ we put

$$
\begin{aligned}
& |\varphi|:=\sup \left\{\left\|\varphi\left(x_{1}, \cdots, x_{p}\right)\right\|: x_{\imath} \in E \text { such that }\left\|x_{\imath}\right\| \leqq 1\right\}, \\
& \|u\|:=\sup \{\|u(x)\|: x \in E \text { such that }\|x\| \leqq 1\} .
\end{aligned}
$$

Obviously $\varphi \mapsto|\varphi|$ resp. $u \mapsto\|u\|$ is a pseudo-norm on $\operatorname{Mult}_{p}(E, F)$ resp. $\operatorname{Hom}_{p}(E, F)$. If $G=(G,\|\cdot\|)$ is a third pseudo-normed $K$-module, then these pseudo-norms satisfy the estimates (2.3.1) and (2.3.2) with $\alpha=1$.

2.6. Let $K=C$ and let $E, F$ be two PO-spaces and $\lambda$ an element of $] 0,1[$. Applying the construction from 2.5 to the semi-normed vector spaces $\left(E,\|\cdot\|_{\lambda}\right)$ and $\left(F,\|\cdot\|_{\lambda}\right)$ over $C$, we obtain a pseudo-norm $|\cdot|_{\lambda}$ resp. $\|\cdot\|_{\lambda}$ on $\operatorname{Mult}_{p}(E, F)$ resp. $\operatorname{Hom}_{p}(E, F)$ for every $p \in N$. We now fix an elencen $\iota \varepsilon$ of $\left.] 10,1\right]$. Ther 


$$
|\cdot|^{0}:=|\cdot|^{0, \varepsilon}:=\sup \left\{|\cdot|_{\lambda}: \lambda \in[1-\varepsilon, 1[\}\right.
$$

resp.

$$
\|\cdot\|^{0}:=\|\cdot\|^{0, \varepsilon}:=\sup \left\{\|\cdot\|_{\lambda}: \lambda \in[1-\varepsilon, 1[\}\right.
$$

is a pseudo-norm on $\operatorname{Mult}_{p}(E, F)$ resp. $\operatorname{Hom}_{p}(E, F)$ again for every $p$. A formal power series $u=\Sigma_{p} u_{p}$ from $F \llbracket E \rrbracket$ is called strictly convergent (with respect to $\varepsilon$ ), if $u$ is convergent with respect to $\|\cdot\|^{0}$. In this case $u$ induces for every element $\lambda$ of $\left[1-\varepsilon, 1\left[\right.\right.$ in a natural way a convergent power series $\bar{u}_{\lambda}=\Sigma_{p}\left(\bar{u}_{p}\right)_{\lambda}$ from $\bar{F}_{\lambda} \llbracket \bar{E}_{\lambda} \rrbracket$.

2.7. Now we introduce a construction, which integrates into the frame given by 2.2 and 2.3 , but which is not a special case of 2.5 .

Let $E, F$ be two PO-spaces, $p \in N_{+}$and let $\lambda, \lambda^{\prime}$ be two elements of $] 0,1[$ such that $\lambda^{\prime} \leqq \lambda$. For a form $\varphi$ from $\operatorname{Mult}_{p}(E, F)=\operatorname{Mult}_{C, p}(E, F)$ resp. a polynomial $u$ from $\operatorname{Hom}_{p}(E, F)=\operatorname{Hom}_{C, p}(E, F)$ let $|\varphi|_{\left(\lambda, \lambda^{\prime}\right)}$ resp. $\|u\|_{\left(\lambda, \lambda^{\prime}\right)}$ be the supremum of the numbers $\left\|\varphi\left(x_{1}, \cdots, x_{p}\right)\right\|_{\lambda^{\prime}}$ resp. $\|u(x)\|_{\lambda^{\prime}}$, where $x_{i}$ resp. $x$ runs through the set of elements of $E$ such that $\left\|x_{i}\right\|_{\lambda} \leqq 1$ resp. $\|x\|_{\lambda} \leqq 1$. Then $|\cdot|\left(\lambda, \lambda^{\prime}\right)$ resp. $\|\cdot\|_{\left(\lambda, \lambda^{\prime}\right)}$ is a pseudo-norm on $\operatorname{Mult}_{p}(E, F) \operatorname{resp.} \operatorname{Hom}_{p}(E, F)$. If $\varepsilon$ is a fixed element of $] 0,1]$, then

resp.

$$
|\cdot|^{1}:=|\cdot|^{1, \varepsilon}:=\sup \left\{\left(\lambda-\lambda^{\prime}\right)^{p-1}|\cdot|\left(\lambda, \lambda^{\prime}\right): \lambda, \lambda^{\prime} \in\left[1-\varepsilon, 1\left[, \lambda^{\prime}<\lambda\right\}\right.\right.
$$

$$
\|\cdot\|^{1}:=\|\cdot\|^{1, \varepsilon}:=\sup \left\{\left(\lambda-\lambda^{\prime}\right)^{p-1}\|\cdot\|_{\left(\lambda, \lambda^{\prime}\right)}: \lambda, \lambda^{\prime} \in\left[1-\varepsilon, 1\left[, \lambda^{\prime}<\lambda\right\}\right.\right.
$$

is a pseudo-norm on $\operatorname{Mult}_{p}(E, F)$ resp. $\operatorname{Hom}_{p}(E, F)$ again. For $y$ from $\operatorname{Mult}_{0}(E, F)$ $=\operatorname{Hom}_{0}(E, F)=F$ we put $|y|^{1}:=\|y\|^{1}:=\|y\|^{0}$. One can show that these pseudonorms satisfy the estimates (2.3.1) and (2.3.2) with $\alpha=2$. A power series $u$ from $F \llbracket E \rrbracket$ is called convergent of type $(1 ;-1)$ (with respect to $\varepsilon$ ), if $u$ is convergent with respect to the pseudo-norms $\|\cdot\|^{1, \varepsilon}$. For example, a power series which is strictly convergent in the sense of 2.6 is convergent of type $(1 ;-1)$.

2.8. For fixed $\varepsilon$ from $] 0,1]$, we denote by $\left(\mathrm{Con}^{\varepsilon}\right)$ the category consisting of the punctured PO-spaces, the morphisms being those formal maps $u:(E, 0) \rightarrow(F, 0)$, which are convergent of type $(1 ;-1)$ with respect to $\varepsilon$. If $\varepsilon=1 / 2$, we write (Con) instead of $\left(\operatorname{Con}^{\varepsilon}\right)$. A morphism $u:(E, 0) \rightarrow(F, 0)$ in $\left(\operatorname{Con}^{\varepsilon}\right)$ will be called direct, if its tangent map $\mathrm{T}(u): E \rightarrow F$ splits in $\mathrm{PO}_{\varepsilon}(\boldsymbol{C})$.

2.9. Let $K$ be a commutative $C$-algebra, $E_{1}, \cdots, E_{k}, F$ a sequence of $K$ modules, and put $E:=E_{1} \times \cdots \times E_{k}$. We suppose that for every $\nu$ from $N^{k}$ we are given a pseudo-norm $\|\cdot\|_{\nu}$ on $\operatorname{Hom}_{K, \nu}(E, F)$. If $t$ is a $k$-tuple from $\left(\boldsymbol{R}_{+}^{*}\right)^{k}$ and $u=\sum_{\nu \in N k} u_{\nu}$ a formal power series from $F \llbracket E \rrbracket$, we put

$$
\|u\|_{t}:=\sum_{\nu \in N}\left\|u_{\nu}\right\| t^{\nu}
$$


Of course $u$ is called convergent with respect to the pseudo-norms $\|\cdot\|_{\nu}$, if there exists a $t$ such that $\|u\|_{t}<\infty$.

2.10. Let $E_{1}, \cdots, E_{k}, F$ be a sequence of PO-spaces, $E:=E_{1} \times \cdots \times E_{k}$ and let $\delta=\left(\delta_{1}, \cdots, \delta_{k}\right)$ be an element of $\{ \pm 1\}^{k}$ and $\nu$ a $k$-tuple from $N^{k}$ such that $|\nu| \geqq 1$. Further let $\lambda, \lambda^{\prime}$ be two elements of $] 0,1\left[\right.$ with $\lambda^{\prime} \leqq \lambda$, and let $\left(\lambda_{1}, \cdots, \lambda_{k}\right)$ denote the tuple given by $\lambda_{i}:=\lambda$ resp. $\lambda_{i}:=\lambda^{\prime}$ if $\delta_{i}=1$ resp. $\delta_{i}=-1$. For a homogeneous polynomial $u$ from $\operatorname{Hom}_{\nu}(E, F)=\operatorname{Hom}_{C, \nu}(E, F)$ let $\|u\|_{\delta,\left(\lambda, \lambda^{\prime}\right)}$ be the supremum of the numbers $\|u(x)\|_{\lambda^{\prime}}$, where $x=\left(x_{1}, \cdots, x_{k}\right)$ is an arbitrary element of $E=E_{1} \times \cdots \times E_{k}$ such that $\left\|x_{\imath}\right\|_{\lambda_{i}} \leqq 1$ for $1 \leqq i \leqq k$. Then $\|\cdot\|_{\partial,\left(\lambda, \lambda^{\prime}\right)}$ is a pseudo-norm on $\operatorname{Hom}_{\nu}(E, F)$. If $\varepsilon$ is a fixed number from $\left.] 0,1\right]$, then

$$
\|\cdot\|_{\delta}^{1}:=\|\cdot\|_{\delta^{\prime}}^{1, \varepsilon}:=\sup \left\{\left(\lambda-\lambda^{\prime}\right)^{|\nu|-1}\|\cdot\|_{\delta,\left(\lambda, \lambda^{\prime}\right)}: \lambda, \lambda^{\prime} \in\left[1-\varepsilon, 1\left[, \lambda^{\prime}<\lambda\right\}\right.\right.
$$

is a pseudo-norm on $\operatorname{Hom}_{\nu}(E, F)$ again. For $\nu=0$ let $\|\cdot\|_{\delta}^{1}:=\|\cdot\|_{\delta}^{1, s}:=\|\cdot\|^{1}$ be the pseudo-norm on $\operatorname{Hom}_{0}(E, F)=F$ defined in 2.7. A formal power series $u=$ $\sum_{\nu \in N^{k}} u_{\nu}$ from $F \llbracket E \rrbracket$ is called convergent of type $(\delta ;-1)$ (with respect to $\varepsilon$ ), if $u$ is convergent with respect to the pseudo-norms $\|\cdot\|_{\delta}^{1}$ (in the sense of 2.9). Note that $u$ is then in particular convergent of type $(1 ;-1)$ in the sense of 2.7 . If $u$ is convergent of type $(\delta ;-1)$, then $u$ induces for every pair $\lambda, \lambda^{\prime}$ of elements of $\left[1-\varepsilon, 1\left[\right.\right.$ with $\lambda^{\prime}<\lambda$ in a natural way a convergent power series $\bar{u}_{\left(\lambda, \lambda^{\prime}\right)}$ from $\bar{F}_{\lambda^{\prime}} \llbracket\left(\bar{E}_{1}\right)_{\lambda_{1}} \times \cdots \times\left(\bar{E}_{k}\right)_{\lambda_{k}} \rrbracket$.

Remark 2.11. In an earlier version of this paper we worked with a weaker notion of convergence for formal maps of PO-spaces. The notion used here was suggested by S. Kosarew.

\section{$\S 3 . \quad$ PO-Lie Groups}

3.1. Let $G=(G, 0)$ be a formal group over $\mathbb{C}$ in the sense of 1.3 such that $G$ is a PO-space. $G$ will be called a PO-Lie group, if the formal maps $m_{G}$ and $j_{G}$ are convergent (with respect to $\varepsilon=1 / 2$ ).

Let now $G$ be a PO-Lie group, $\omega: G \times(E, 0) \rightarrow(E, 0)$ a convergent operation of $G$ on a punctured PO-space $(E, 0)$ and $\varepsilon$ an element of $] 0,1 / 2]$. We say that $\omega$ is direct (with respect to $\varepsilon$ ), if the corresponding orbital map $G \rightarrow(E, 0)$ is a direct morphism in $\left(\mathrm{Con}^{\varepsilon}\right)$.

Example 3.2. Let $r$ be a tuple from $\left(\mathbb{R}_{+}^{*}\right)^{n}$ and $U:=P(0 ; r) \leqq \mathbb{C}^{n}$ the open polycylinder of polyradius $r$ with center 0 and $G:=\Gamma\left(U, \mathcal{O}_{C n}\right)^{n}$. Further let

$$
m=m_{G}:(G, 0) \times(G, 0) \longrightarrow(G, 0)
$$

be the formal map with series expansion $m=\sum_{p, q \in N} m_{p, q}$ given by $m_{p, 1}(e, f):=$ $(1 / p !) D^{p}(f) \cdot e^{p}$ if $p \geqq 0, n_{1,0}(e, f):=e$ and $m_{p, q}:=0$ otherwise. Then $G$ is a 
formal group over $C$ with respect to $m_{G}$. S. Kosarew has verified that $m_{G}$ and $j_{G}$ are convergent in the sense of 2.7. Hence $G$ is even a PO-Lie group.

3.3. Let $E$ be a PO-space and $t_{0}$ an element of $[0,1[$. Then we denote by

$$
(E, 0)_{t_{0}}^{2}:(\mathrm{Gan}) \longrightarrow(\text { sets })
$$

the functor on the category (Gan) of germs of (finite dimensional) analytic spaces, sending a germ $S=(S, 0)$ to the $\mathcal{O}_{S, 0}$-module

$$
(E, 0)_{t_{0}}(S):=\lim _{\lambda>t_{0}} \operatorname{Hom}\left(S,\left(\bar{E}_{\lambda}, 0\right)\right) \text {; }
$$

here $\operatorname{Hom}\left(S,\left(\bar{E}_{\lambda}, 0\right)\right)$ is the set of analytic map-germs $f: S \rightarrow \bar{E}_{\lambda}$. such that $f(0)$ $=0$. Let now $F$ be a second PO-space and $u:(E, 0) \rightarrow(F, 0)$ a morphism in $\left(\mathrm{Con}^{1-t_{0}}\right)$. Then $u$ obviously induces a morphism

$$
\check{u}_{t_{0}}:(E, 0)_{t_{0}} \longrightarrow(F, 0)_{t_{0}}
$$

of functors. Since $(F, 0)_{t_{0}}(\operatorname{Spec}(C))$ consists of exactly one element, the fibre of $\check{u}_{t_{0}}$ is a well defined subfunctor $W_{0^{t}}^{\check{t}}$ of $(E, 0)_{t_{0}}$.

3.4. Let now $G$ be a PO-Lie group and $t_{0}$ be a fixed element of $] 1 / 2,1[$. Then $G_{t_{0}}^{\check{2}}:=(G, 0) \check{t}_{0}$ is obviously a group-valued functor on (Gan). If $\omega: G \times(E, 0) \rightarrow(E, 0)$ is a convergent operation of $G$ on a punctured PO-space $(E, 0)$, then $\check{\omega}_{t_{0}}$ is an operation of the group-valued functor $G_{t_{0}}$ on the setvalued functor $(E, 0)_{t_{0}}^{2}$.

Let $\eta: G \times(F, 0) \rightarrow(F, 0)$ be another convergent operation and let $u:(E, 0) \rightarrow$ $(F, 0)$ be a $G$-equivariant convergent map. Then the functor morphism $\check{u}_{t_{0}}$ is obviously $G_{t_{0}}$-equivariant. If $G$ operates with fixed point on $(F, 0)$, then the fibre $W_{t_{0}}$ of $\check{u}_{t_{0}}$ is invariant under the operation of $G_{z_{0}}^{\check{ }}$,

\section{$\S 4$. The Quotient Theorem}

4.1. Let $F: \mathcal{C} \rightarrow$ (sets) and $G: \mathcal{C} \rightarrow$ (groups) be contravariant functors on a category $\mathcal{C}$, and suppose given an operation of $G$ on $F$. Then the quotient groupoid $F / G \stackrel{p}{\rightarrow} \mathcal{C}$ is defined as follows. The objects of $F / G$ are the elements $a$ of $F(S)$ with $S \in C$. If $a^{\prime} \in F\left(S^{\prime}\right)$ and $a \in F(S)$ are two objects, then $\operatorname{Hom}_{F / G}\left(a^{\prime}, a\right)$ is the set of pairs $(f, g)$ from $\operatorname{Hom}_{C}\left(S^{\prime}, S\right) \times G\left(S^{\prime}\right)$ such that $g \cdot F(f)(a)=a^{\prime}$. Obviously $(\overline{F / G})(S)=F(S) / G(S)$ is the orbit space of $F(S)$ with respect to the operation of $G(S)$.

4.2. Suppose now given a PO-Lie group $G$ operating on two punctured POspaces $(E, 0)$ and $(F, 0)$ such that $G$ acts with fixed point on $(F, 0$. Further let $u:(E, 0) \rightarrow(F, 0)$ be a strictly convergent $G$-equivariant morphism in (Con) 
and $t_{0}$ an element of $\left[1 / 2,1\left[\right.\right.$. Then the group valued functor $G_{t_{0}}$ operates on the fibre $W_{t_{0}}$ of $\check{u}_{t_{0}}$, and the corresponding quotient groupoid $W_{t_{0}}^{\check{r}} / G_{t_{0}}$ over (Gan) satisfies Schlessinger's condition $\left(\mathrm{S}^{\prime}\right)$, see [1]. The following theorem gives a criterion for $W_{t_{0}}^{\check{r}} / \check{G}_{t_{0}}$ to have a semiuniversal deformation.

Theorem 4.3. Let the assumptions and notations be as in 4.2. Moreover suppose that the following conditions hold:

(1) The operations $G \times(E, 0) \stackrel{\omega}{\rightarrow}(E, 0)$ and $G \times(F, 0) \rightarrow(F .0)$ are convergent of type $(-1,1 ;-1)$, and $E, F$ are $\left(1-t_{0}\right)$-good.

(2) $\omega$ is direct with respect to $1-t_{0}$.

(3) $u$ is direct with respect to $1-t_{0}$.

(4) The tangent space $\left.\overline{\left(W_{t_{0}}^{\vee} / G_{t_{0}}\right.}\right)(D)$ is finite dimensional. ${ }^{1)}$

Then the groupoid $W_{t_{0}} / \check{G}_{t_{0}}$ has a semiuniversal deformation.

Sketch of proof. By (3), the image $I$ of $\mathrm{T}(u): E \rightarrow F$ is a direct summand of $F$ in $\mathrm{PO}_{1-t_{0}}(\boldsymbol{C})$. Hence we can find a retraction $v: F \rightarrow I$ onto $I$ in $\mathrm{PO}_{1-t_{0}}(\boldsymbol{C})$. For an element $\lambda$ of $\left[t_{0}, 1\left[\right.\right.$ let $W_{\lambda}$ resp. $M_{\lambda}$ denote the fibre of $\vec{u}_{\lambda}:\left(\bar{E}_{\lambda}, 0\right) \rightarrow$ $\left(\bar{F}_{\lambda}, 0\right)$ resp. $\bar{v}_{\lambda} \bar{u}_{\lambda}:\left(\bar{E}_{\lambda}, 0\right) \rightarrow\left(\bar{I}_{\lambda}, 0\right)$. Then $W=\left(W_{\lambda}\right)_{\lambda \in] t_{0}, 1[}$ resp. $M=\left(M_{\lambda}\right)_{\lambda \in] t_{0}, 1[}$ is a direct system of germs of Banach analytic spaces resp. manifolds suct that $W \leqq M$ and $\mathrm{T}\left(W_{\lambda}\right)=\mathrm{T}\left(M_{\lambda}\right)$ holds for all $\lambda^{2)}$. Moreover we have $W_{t_{0}}(S)=$ $\varliminf_{\lambda>t_{0}} \operatorname{Hom}\left(S, W_{\lambda}\right)$ for any germ $S$ from (Gan). By (4), there exists an index $\lambda>t_{0}$ such that the canonical map from $\mathrm{T}\left(W_{\lambda}\right)$ to $\left(\overline{W_{t_{0}}^{\sim} / G_{t_{0}}^{\sim}}\right)(D)$ is surjective. We choose a finite dimensional smooth subgerm $N \subseteq M_{\lambda}$ for which the map

$$
\mathrm{T}(N) \longrightarrow\left(\overline{W_{t_{0}}^{2} / G_{t_{0}}^{2}}\right)(D)
$$

is bijective. Then $Y:=N \cap W_{\lambda}$ is a subgerm such that $\mathrm{T}(Y)=\mathrm{T}(N)$. Moreover $Y$ is finite dimensional by [7], 7.5, Prop. 7 .

Let $\lambda^{\prime}$ be an arbitrary index such that $t_{0}<\lambda^{\prime}<\lambda$. Then by $(1) \bar{\omega}_{\left(\lambda, \lambda^{\prime}\right)}$ induces an analytic map-germ from $\left(\bar{G}_{\lambda^{\prime}}, 0\right) \times W_{\lambda}$ into $W_{\lambda^{\prime}}$. Now one shows, using (2) and the propositions 4.4, 4.5 stated below combined with the inverse mapping theorem for analytic maps of Banach analytic manifolds, that the morphism

$$
\left(\bar{G}_{\lambda^{\prime}}, 0\right) \times Y \stackrel{\bar{\omega}_{\left(\lambda, \lambda^{\prime}\right)}}{\longrightarrow} W_{\lambda^{\prime}}
$$

is smooth. From this one can easily conclude that the canonical functor $\rho: \dot{Y} \rightarrow W_{t_{0}}^{\check{2}} / G_{t_{0}}^{\check{ }}$ is minimally $\operatorname{smooth}^{3)}$. Hence $\rho\left(\mathrm{id}_{Y}\right) \in\left(W_{t_{0}}^{\check{2}} / G_{t_{0}}^{\sim}\right)(Y)$ is a semiuniversal deformation of $W_{t_{0}}^{\check{r}} / G_{t_{0}}$.

1) Here $D:=\operatorname{Spec}(\mathbb{C}[\varepsilon])$ is the double point.

2) For a germ $Z$ of a (Banach) analytic space $\mathrm{T}(Z)$ denotes the tangent space in the distinguished point.

3) If $Z$ is a germ of an analytic space, $Z$ denotes she functor on (Gan) defined by $Z$. 
In the above proof, we made use of the following two propositions. Detailed proofs are given in [5], (II 12).

Lemma 4.4. Let $X$ be a finite dimensional complex space, let $i: F \rightarrow G$ be an injective continuous linear map of Banach spaces and let $f: X \rightarrow F$ be a holomorphic map. Then the subspaces $f^{-1}(0)$ and $(i \circ f)^{-1}(0)$ of $X$ coincide.

Lemma 4.5. Let $X \rightarrow S$ be a map of germs of Banach analytic spaces of finite relative dimension, and let $Y \cong X$ be a subgerm of $X$ which is S-anaflat. ${ }^{4)}$ Then if $X_{0}=Y_{0}$ holds for the fibres in the distinguished point $0 \in S$, we already have $X=Y$.

Remarks 4.6. (1) The proof of 4.3 presented here uses in an essential way the theory of Banach analytic spaces in the sense of Douady. In a forthcoming paper I will show that one can prove 4.3 using only power series techniques. As a byproduct, such a "finite dimensional" proof also gives more precise information on the structure of the base $Y$ of the semiuniversal deformation of the quotient groupoid $W_{t_{0}}^{r} / \check{G}_{t_{0}}$.

(2) One can also show a relative version of the quotient theorem 4.3. Again, I will provide the details at another place.

\section{References}

[1] Bingener, J., Offenheit der Versalität in der analytischen Geometrie, Math. Z., 173 (1980), 241-281.

[2] - Splitting theorems for PO-complexes, Publ. RIMS, Kyoto Univ., 23 (1987), 527-534.

[3] - Local moduli spaces in analytic geometry, Publ. RIMS, Kyoto Univ., 23 (1987), 543-552.

[4] - Local moduli for strictly pseudoconvex spaces, Publ. RIMS, Kyoto Univ., 23 (1987), 553-558.

[5] Bingener, J. and Kosarew, S., Lokale Modulräume in der analytischen Geometrie, Vieweg-Verlag, Braunschweig/Wiesbaden (1987).

[6] Donin, I.F., Complete families of deformations of germs of complex spaces, Math. USSR Sb., 18 (1972), 397-406.

[7] Douady, A., Le probleme des modules pour les sous-espaces analytiques compacts d'un espace analytique donné, Ann. Inst. Fourier, 16 (1966), 1-95.

4) In the sense of [7]. 